\title{
Die Vermessung der Klinik
}

\section{A Survey of the clinic}

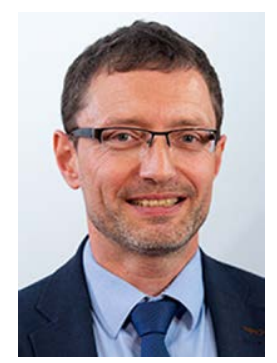

Eugen Feist

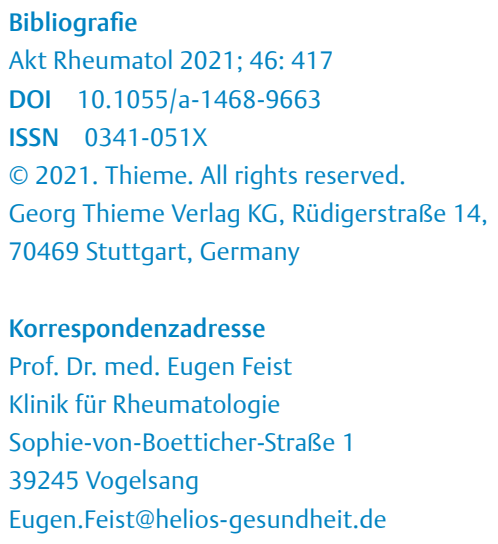

Vielen von uns mag es ähnlich ergehen, wenn wir abends die Klinik verlassen oder unsere Praxistür nach einem langen anspruchsvollen Arbeitstag abschließen. Wir machen uns auf den Heimweg und resümieren dabei gelegentlich: „Was war das doch für ein verrückter Tag?!“ oder „Was habe ich heute eigentlich alles weggearbeitet?!“.

Zumindest für die in der Klinik tätigen Kollegen unter uns kann die Publikation von Leonore Unger mit Koautoren in dieser Ausgabe der Aktuellen Rheumatologie eine gewisse Orientierung geben. Erstmals hat Sie mit Ihren Kollegen unglaublich akribisch die ärztliche Arbeitszeit in einer internistischen Klinik mit rheumatologisch-nephrologischem Profil erfasst. Das Ergebnis kann uns erstaunen lassen, mit wie viel weniger Personal wir eigentlich den Alltag üblicherweise meistern. Es kann aber auch ein ernsthaftes Argument dafür sein, über einen notwendigen ärztlichen Personalschlüssel nicht nur nachzudenken, sondern diesen auch einzufordern. Wie oberflächlich das DRG System dabei gerade die Leistung einer rheumatologischen Klinik heutzutage bewertet, ist uns allen zumindest unterschwellig bewusst. Mit dieser Publikation haben wir ein Argument mehr um zu belegen, dass unsere Leistungen tatsächlich nicht adäquat abgebildet sind.

Die Arbeit zeigt aber auch die unglaublich vielseitige Welt der Rheumatologie allein schon mit einem Blick in die Tabelle 2, die die Hauptdiagnosen des Überwachungszeitraum eines einzigen Monats aufführt. Das was wir hier aufgeführt sehen, veranschaulich wie differenzialdiagnostisch breit wir aufgestellt sein müssen und wie hoch letztlich auch die Anforderungen an unser ständig zu aktualisierendes Wissen sind. Liebe Kolleginnen und Kollegen, Sie wissen, der Arbeitsalltag endet nicht mit dem eingangs beschriebenen Moment des Resümierens, sondern geht in aller Regel am Abend und Wochenende weiter. Schauen Sie doch bitte in diesem Moment kurz auf Kalender und Uhr. Sie sind vermutlich gerade dabei in Ihrer „Freizeit“ diese Ausgabe der Aktuellen Rheumatologie zu lesen.

Das sollte jedoch nicht dazu führen, dass Sie das Heft gleich wieder zuschlagen, denn wir haben noch weitere interessante Artikel für sie bereit. Im gerade angestimmten Tenor hat die Rheumatologie ein Date mit der Hepatologie im Beitrag von Alexander Zipprich zur Diagnostik und Therapie von autoimmunen Lebererkrankung. Hier geht es um die drei hepatologischen Klassiker, die uns so oft in Überlappung mit rheumatischen Grunderkrankungen begegnen. Eine zweifelsohne lohnende Lektüre, die in prägnanter Form alle wichtigen Infos bereithält und u. a. auch neue Therapieoptionen für die refraktäre PBC anspricht.

In ihrer Promotionsarbeit hat Helene von Bremen aus meiner Arbeitsgruppe Immunoassays zum Nachweis von Anti-Drug Antikörpern gegen Infliximab und korrespondierenden Medikamentenspiegel evaluiert. Ich denke, eines können wir daraus schlussfolgern: Die Methodik ist soweit. Wir können, wenn wir wollten, ein therapeutisches Drugmonitoring auch in der Praxis standardisiert anbieten.

Doch nicht alles ist leicht umsetzbar und ein „Home Run“, wie wir aus Erfahrungen von Norman Best und Dana Loudovici-Krug zur Planung einer Studie in der Versorgungsforschung lernen. Schließt sich hier der Kreis? Sind wir an einem Punkt angelangt, an dem wir es einfach nicht mehr leisten können noch mehr zusätzlich nebenbei zu erledigen? Laufen wir Gefahr in der Routine komplett unterzugehen, können wir es uns zeitlich schlicht nicht mehr leisten, in der Praxis wissenschaftlich zu arbeiten und andere Studien zu unterstützen? Die Autoren kommen zum Fazit, dass eine Überzeugungsarbeit vor Beginn der Datenaufnahme entscheidend ist. Das ist ohne Frage richtig. Doch hohe Qualität in Klinik und Forschung ist letztlich nur bei einer guten Personalsituation und zukünftig nur bei ausreichend rheumatologischem Nachwuchs zu erreichen. Also biegen Sie doch bitte noch auf dem Nachhauseweg kurz in den Studententreff ab oder gehen zu einer Weiterbildung für Assistenzärzte und verkünden, dass Rheumatologen mit die glücklichsten Ärzte mit toller Work-Life Balance sind!

Herzliche Grüße

Ihr Eugen Feist 